\title{
Digital Interactive Kanban Advertisement System Using Face Recognition Methodology
}

\author{
Feng-Yi Cheng, Chu-Ja Chang, Gwo-Jia Jong \\ Department of Electronics Engineering, National Kaohsiung University of Applied Sciences, Kaohsiung. \\ Email: changchuja@gmail.com
}

Received May, 2013

\begin{abstract}
Most of advertisement systems are presently still launch the publicity content by the static words and pictures. Recently, this static advertisement model will not be able to attract people's attention more and more. Moreover, the static information content of advertisement system is limited because of the layout shown size. It can not also fully demonstrate the information content of advertisement system. In this paper, we develop a digital interactive kanban advertisement system using face recognition methodology to solve these problems. The system captures the person's face through the camera. The digital advertisement content size is relevant by the person and camera observation locations. In this paper, we adopt the Adaboost algorithm to judge people face, and the system only need to grab the position of the face. The system doesn't built expensive and complex equipment to reduce the system cost and enhance the system performance. This system can also achieve the same similar digital interactive advertising effectiveness.
\end{abstract}

Keywords: Face Recognition; Kanban Advertisement; Adaboost; Interactive

\section{Introduction}

There are various kinds of advertising media now. For example: televisions, broadcastings, magazines, newspapers, outdoor advertising and transit advertising...etc. Beside above media, the appearance of the web advertising makes advertising having more and more development space. An advantage of web advertising is that consumers can select advertising freely and obtain messages advertising transmit immediately. Advertising can interact with consumers and transmit feedback instantly, but traditional advertising can't [1,2]. In this paper, interactive multimedia as the theme to explore the visual interface design and interactive multimedia development, according to the popular trend of today's interactive multimedia authoring the common interactive multimedia such as: interactive web pages, DVD movies menu, web advertising, teaching CD-ROM...etc., because of the rapid growth of technology and the technology matures, the traditional static advertising, web, gradually interactive multimedia replaced [3].

In recent years, face detection technology tends to mature, the technology has been widely used in cameras, computer identification system and interactive advertising. In [4], they research proposes a system that engages audience to the advertisement through interactive applications and provides data to the advertiser/producer about their audience, but we think that is too complex.
Therefore, we propose an interactive system, using text and pictures to do the interactive display, it can allow users to quickly understand the contents of advertisements.

\section{Interactivity Kanban Advertisement}

The Figure 1 is the configuration of interactive kanban advertisement system. At first, we capture the camera image, then color segmentation extracts the skin color of a face from a cluttered image; then, binary imaging further forms a more complete region. Next, morphological erosion eliminates some of the small spots in a tested image. Contrary to erosion, dilation enlarges and connects a small and disconnected, but marked, facial region. Subsequently, connected component labeling is employed to mark multiple faces in the image. Finally, an area threshold and an aspect ratio are used to validate the corrected facial region. After then we use the Adaboost algorithm [7-10] to make face recognition. At last, we can judge by the distance between the captured face and advertising, when the person is closer and closer, the words will accord to the distance for scaling to achieve

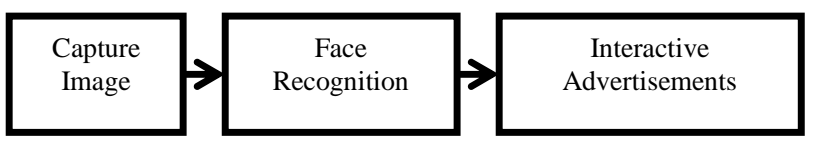

Figure 1. Interactivity kanban ad system block diagram. 
the purpose of attracting users.

The Figure 2 is our hardware configuration of capture image, the camera mounts on the Kanban Advertisement above.

\section{Face Recognition}

The Figure 3 is our face recognition system, the face recognition has three processing steps: Skin color detection, Detect face region, facial feature points and finally, through Adaboost screening the most right face of people. This part is mainly to capture each person's face form the image.

\subsection{Skin Color Detection}

The color segmentation is an important pre-processing step in the face recognition methods. We are used HIS method [5] to detection face skin color. Frist, we transform the image of RGB three color changes to $\mathrm{H}$, S. It can be written as

$$
H=\left\{\begin{array}{cc}
\theta, & \text { if } B \leq G \\
360-\theta & \text { if } B>G
\end{array}\right.
$$

where

$$
\begin{aligned}
& \theta=\cos ^{-1}\left\{\frac{\frac{1}{2}[(R-G)+(R-B)]}{\left[(R-G)^{2}+(R-B)(G-B)\right]^{1 / 2}}\right\} \\
& S=1-\frac{3}{(R+G+B)}[\min (R, G, B)]
\end{aligned}
$$

Then we can follow the rule to find skin color $f_{c}$ :

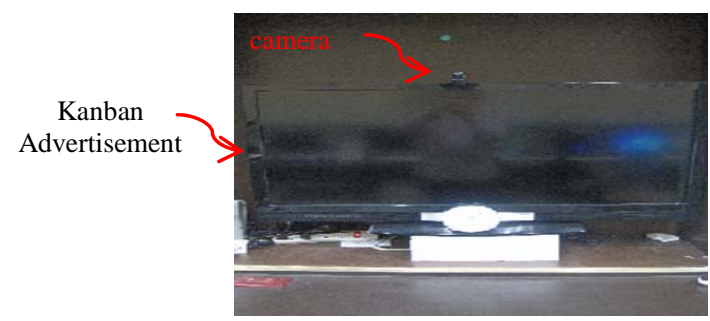

Figure 2. Interactivity kanban ad system configuration.

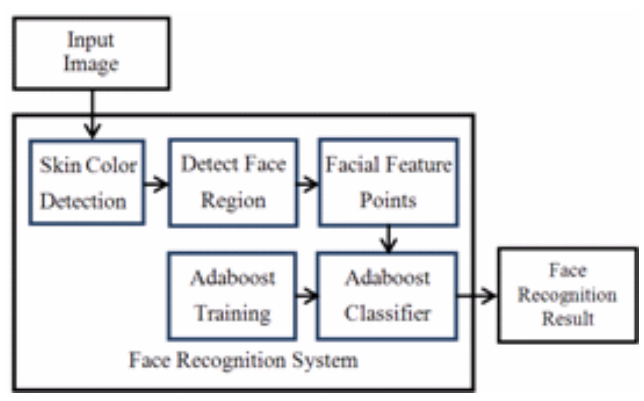

Figure 3. Face recognition block diagram.

$$
f_{c}=\left\{\begin{array}{l}
1, \text { if } 8 \leq H \leq 32 \text { and } 30 \leq S \leq 163 \\
0, \text { otherwise }
\end{array}\right.
$$

After the skin color detection, we only see the portion of skin color, as shown in Figure 4.

\subsection{Face Region Detection Method}

After the Skin color detection, we change the RBG to binary used thresholding. Then, we consulted the paper's method [6] to the binary image is sub-divided into blocks. Then, the total skin area within a block is computed, and if this is greater than or equal to $40 \%$ of the block area, the block label is assigned to be skin. A connected region step is then performed by examining the 8 neighbourhood connectivity among the blocks to create a set of candidate regions. Face regions are selected amongst the candidate regions as the regions having an aspect ratio corresponding to the $1.2-2.0$ ratio, than use the rate value of the image to define the threshold. It is showed in Figure 5.

\subsection{Capture Facial Feature Points}

\subsubsection{Eyes Detection}

It is obvious that eyes are non-skin color regions, the $C_{r}$ and $C_{b}$ component of eyes and skin contains bigger difference in the $Y C_{r} C_{b}$ space, and the $C_{b}$ is higher than the $C_{r}$ in the eyes region. It can detection location and size by above information.

\subsubsection{Mouths Detection}

Mouth is also non-skin color region, in the mouth the $C_{r}$ is much higher and the $C_{b}$ much lower. Increasing the difference between the $C_{b}$ and $C_{r}$ can accurately detected size and location.

\subsection{Adaboost Algorithm}

Machine learning algorithm is flourishing in recent year, widely used at various levels. Face Detection this issue in order to obtain better characteristics also introduces a machine learning concepts, these studies are a breakthrough in the past to the face detection frame, most notably the 2004 study is presented using the integral image Viola for the characteristic value of the AdaBoost face detection method. AdaBoost is an algorithm for constructing a "strong" classifier as linear combination of “weak” classifiers [7-10].

\subsubsection{Haar-Like Features and Integral Image}

A set of Haar-like features, used as the input features to the cascaded classifiers, are shown in Figure 6. In our work, Haar-like features consideration is using integral image to improve computation efficiency. 


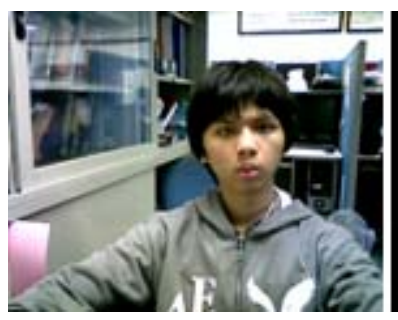

(a)

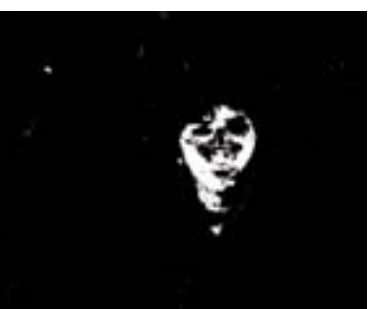

(b)
Figure 4. (a) Original image; (b) Skin color detection.

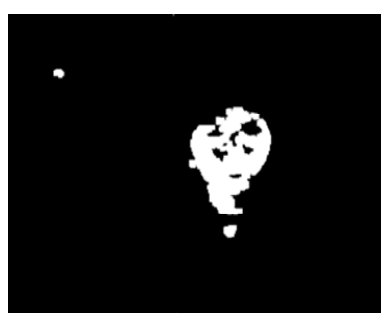

(a)

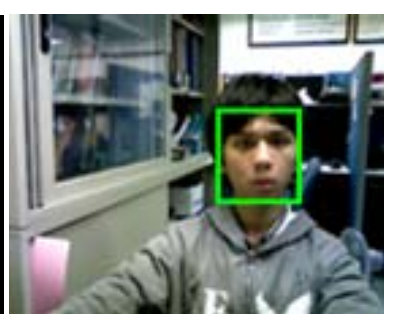

(b)
Figure 5. (a) Morphological process; (b) Face region detection.

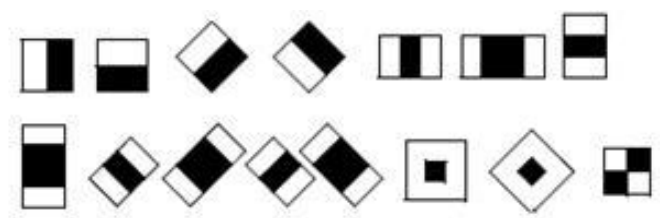

Figure 6. The Haar-like features for AdaBoost algorithm.

The Haar-like features that shown in Figure 6. It used in our face detection system. The features can be rapidly computed at different scales by introducing "Integral Image”.

\subsubsection{AdaBoost Algorithm}

In fact AdaBoost is a classification of concepts, for example, In order we pick a better than normal a little bit $(>=50 \%$ ) of the algorithm, it can again and again use update weighting approach to reduce error rate, The process is as follows

1) Input $M$ sample of the target image and $N$ sample not of the target image, and I search the number of features.

2) Initialize weights

Target image samples weights

$$
W P_{m}=\frac{1}{2 M}
$$

Non-target image samples weights

$$
W N_{n}=\frac{1}{2 N}
$$

3) For each feature $j$, train a weak classifier $T$, and evaluate its error $E$ with respect to $W$

$$
E=\sum_{m=1}^{M} W P_{m}\left(1-T_{m}\right)+\sum_{n=1}^{N} W N_{n} \cdot T_{n}
$$

In this derivation when $T=1$ consistent with the image features, $T=0$ does not meet.

4) Using step 3. Add the choose features to the stage and determine the corresponding weights

$$
W_{i}=\log \left(\frac{1-E}{E}\right)
$$

5) For step 3. Searching the better features to updates image sample weights. Updated target image sample weights.

$$
W P_{m} \leftarrow W P_{m} \cdot\left(\frac{E}{1-E}\right)^{1-E}
$$

Updated non-target image samples weights.

$$
W N_{n} \leftarrow W N_{n} \cdot\left(\frac{E}{1-E}\right)^{1-E}
$$

6) Normalize the weights

$$
\begin{aligned}
& W P_{m} \leftarrow\left(\frac{W P_{m}}{\sum_{m=1}^{M} W P_{m}}\right) \\
& W N_{n} \leftarrow\left(\frac{W N_{n}}{\sum_{n=1}^{n} W N_{n}}\right)
\end{aligned}
$$

7) Check whether the number of the current search features to meet the demand, if the lack of jump back to step 3, otherwise the end of.

\section{Interactive Advertisement System}

The Figure $\mathbf{7}$ is our interactive advertisement system flow process. At first, we capture the camera’s image,

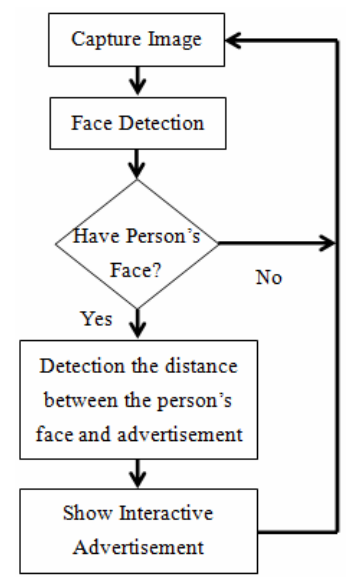

Figure 7. Interactive ad system flow process. 
then thought the image to do face recognition processing, to get the information of people who watch the Advertisement, and then the system further determine whether capture face or not, in other words, to determine if someone is watch the kanban advertisement system, after then to detection the distance between the person's face and advertisement, when people are approached the advertisement, the advertisement will also show more message telling the people, let people can learn more about the details of the advertisement, to increase the impression of people watch advertisement.

\section{Result and Discuss}

We use a 20 million pixels webcam and a 36-inch TV to achieve the Interactive Kanban Advertisement System. The webcam is set in place of $130 \mathrm{~cm}$ high and angle of 90 degrees. The Figure 8 shows the information that the relationship between pixel size of face and the distance from person to camera. When the system captures the person's face, we can use the pixel size of face to determine where the person.

The Figure 9 is the user interface of program, this program of interface can divided into two parts, the camera of image is on the left, the interactive kanban advertisement is on the right. If someone walks past in front of the kanban advertisement, the system will catch person's face, and the kanban advertisement also shows some words to attract people's attention, it is like Figure 9.

When person is closer and closer, the system will calculate the face of pixels to detect the distance, if people rely on close enough, the system will change the adver-

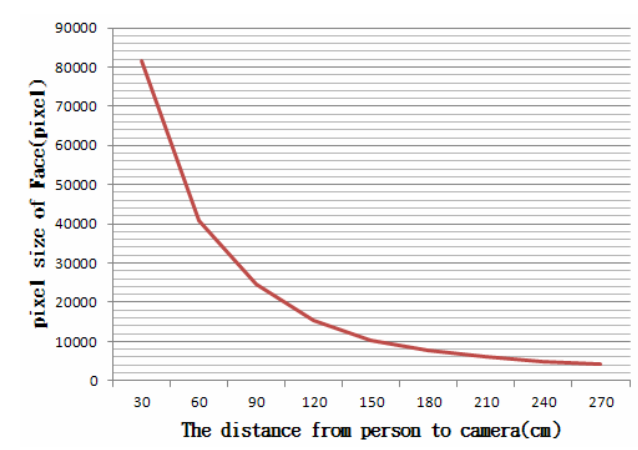

Figure 8. Graph of ratio between person and camera.

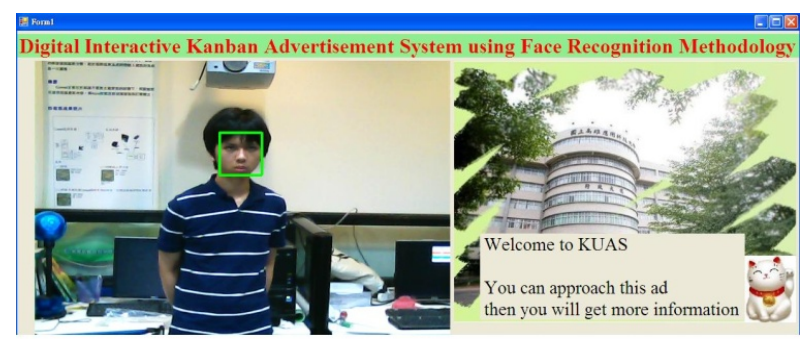

Figure 9. The user interface of program.

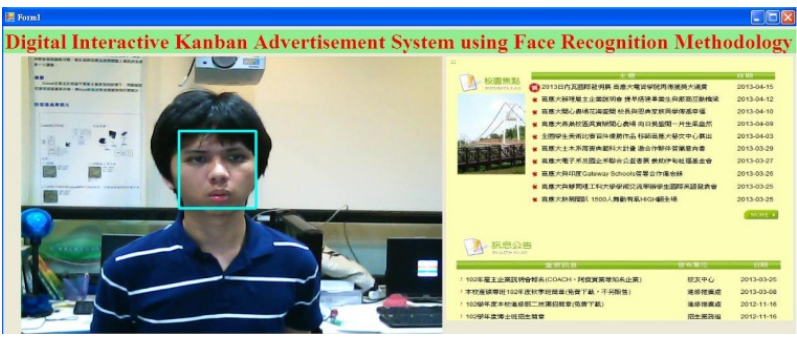

Figure 10. It change the ad content when people rely on close enough.

tising content, display more information attract people to continue to watch, it is like Figure 10.

\section{Conclusions}

In this paper, we propose an Adaboost algorithm approach to the face recognition for applications of interactive advertisement system. Although it has a lot of people to research for the subject so far, however, the proposed approach are more complex, build may more cost or need to take the time to interact with advertisement, caused people inconvenience. Our system only through the distance between the face and advertising to interact, through words and pictures to attract people, reduce the complexity of the system also allows people quickly to understand more information of advertisements.

\section{REFERENCES}

[1] J. H. Cho, Y. J. Sah and J. Ryu, “A New Content-related Advertising Model for Interactive Television,” Broadband Multimedia Systems and Broadcasting 2008, March 31 2008-April 2 2008, pp. 1-9.

[2] M.-H. Hsieh, D.-L. Yang and J.-Y. Dai, “A Face Recognition System Prototype to Evaluate the Effectiveness of Digital Advertisement,” 2010 Conference on Computer Vision, Image Processing and Information Technology, 2010-06. Zhongli, Taiwan, pp. 283-289.

[3] J. Kim and S. Kang, “An Ontology-Based Personalized Target Advertisement System on Interactive TV,” Consumer Electronics (ICCE), 2011 IEEE International Conference, 9-12 Jan. 2011, pp. 895 - 896.

[4] M. Taspinar, A. T. Naskali, M. Kurt and G. Eren, "The Importance of Customized Advertisement Delivery Using 3D Tracking and Facial Recognition,” in Proc. The Second International Conference on Digital Information and Communication Technology and its Applications (DICTAP), 2012, pp. 526-530.

[5] S. Guerfi, J.-P. Gambotto and S. Lelandais, "Implementation of the Watershed Method in the HSI Color Space for the Face Extraction,” Advanced Video and Signal Based Surveillance, Sept. 2005, pp. 282-286.

[6] M. Rahman and N. Kehtarnavaz, "Real-TimeFace-Priorit y Auto Focus for Digital and Cell-Phone Cameras," IEEE Transactions on Consumer Electronics, Vol. 54, No. 4, 2008, pp. 1506-1513.doi:10.1109/TCE.2008.4711194 
[7] J. X. Ruan and J. X. Yin "Multi-Pose Face Detection Using Facial Features and AdaBoost Algorithm," Second International Workshop on Computer Science and Engineering, 2009, pp. 31-34.

[8] Y.-W. Wu and X.-Y. Ai, "Face Detection in Color Images Using AdaBoost Algorithm Based on Skin Color Information," Workshop on Knowledge Discovery and Data Mining, 2008, pp. 339-342.
[9] S. A. Inalou and S. Kasaei “AdaBoost-Based Face Detection in Color Images with Low False Alarm,” Second International Conference on Computer Modeling and Simulation, 2010, pp.101-111. doi:10.1109/ICCMS.2010.287

[10] Y. C. Xing, Z. Z. Wang and W. P. Qiang, "Face Tracking Based Advertisement Effect Evaluation,” Image and Signal Processing, 2009. CISP '09. 2nd, 2009, pp. 1-4. 